\title{
Basic Logic Gate Detector Using Atmega328P and Android App \\ ${ }^{* 1}$ Yash Patil, ${ }^{2}$ Sumedh Naik, ${ }^{3}$ Rashmi Phadnis, ${ }^{4}$ Vijaya Kamble \\ 1-4 Sardar Patel Institute of Technology, Mumbai \\ Email:yashpatil1998@gmail.com, sumedhnaik1598@gmail.com,rashmi.phadnis98@gmail.co,vijaya_kamble@spit.ac.in
}

Received: $3^{\text {th }}$ November 2018, Accepted: $1^{\text {th }}$ February 2019, Published: $30^{\text {th }}$ June $_{2019}$

\begin{abstract}
GATEway is a device which helps in detecting a basic logic gate, the result of which is displayed on a mobile application available for android devices.

Apart from just detecting the basic gate IC, the mobile application will also have a link to a more elaborate description of that IC, which would help the user to gain extra knowledge.
\end{abstract}

\section{Keywords}

Basic Logic Gate, Three Tier Architecture, Companion App.

\section{Introduction}

It has become very important to provide a hands-on learning experience to school and college students to actually understand and learn what is taught, especially in those subjects having hardware involved. Knowledge about hardware cannot only be learned through books. It needs a hands-on approach with heavy emphasis on familiarity of the student with handling the hardware components. At the same time, your practical skills need a strong understanding of the theoretical knowledge of the component. Keeping in mind that both practical and theoretical knowledge are a must and each one has to supplement the other, the need of a device such as GATEway was felt. For students venturing into the world of hardware and digital circuits, having a working knowledge of basic gates is a must. While working with gates, it is essential that you identify the correct gate and have the datasheet for the same. GATEway provides a way to identify the gate without even having to look up the IC number. It also pulls up the datasheet and provides tutorials on the working of the gate on your Android app.

\section{Objective}

The main objective of GATEway is to enforce interest in the working of basic logic gates. This product can detect the basic gate and also help the user to see the working and other related details of the detected gate. The secondary objective is to act as a teaching aid by providing tutorials of the detected gate.

\section{System Design}

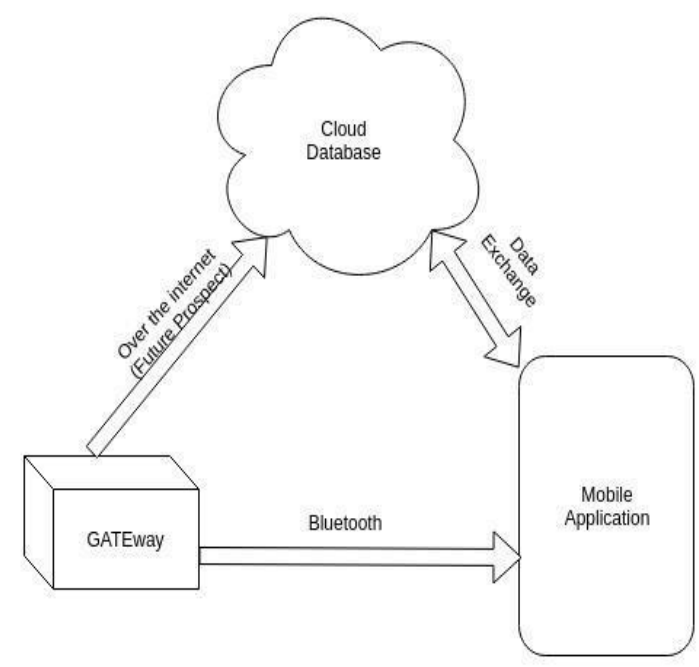

Figure 1: Three Tier Architecture.

Our product GATEway is built on a three tier architecture. A three-tier architecture basically means the working of our product is based on three key blocks. The first block is the actual hardware. This hardware actually detects the basic logic gate which is inserted in the shown slot. The hardware is connected to the second block in this architecture, which is the 
GATEway companion mobile application, via bluetooth. The companion app is connected to the third component in the architecture, which is the cloud server. The future prospects of our product is where all the required necessary documentations and tutorials on using the detected basic gate is displayed on the companion app.

\section{Design Requirements}

Following are the components used in GATEway

Atmega328P Microcontroller

Atmega328P is a low power, 8-bit micro controller based on the AVR RISC architecture. It has 23 programmable I/O lines, with an operating voltage range of $1.8 \mathrm{~V}-5.5 \mathrm{~V}$. Operating temperature is from -40 degrees to 150 degrees.

HC-05 Bluetooth Module

HC-05 Module is a Bluetooth SPP (Serial Port Protocol) Module for transparent wireless serial connection setup. It uses 3 Mbps modulation and has a radio transciever and baseband of frequency $2.4 \mathrm{GHz}$.

LM7805 Voltage Regulator

LM7805 voltage regulator takes an input voltage range from $7 \mathrm{~V}-35 \mathrm{~V}$ and gives a constant output of $5 \mathrm{~V}$. It has a current rating of $1 \mathrm{~A}$.

$16 \mathrm{MHz}$ Crystal Oscillator

Atmega328P requires a clock source of $16 \mathrm{MHz}$. The output of the crystal oscillator is fed as input to the Atmega328P Micro-controller as its clock.

16 and 28 pin IC Base

The 16 and 28 pin IC bases are used to mount ICs of pins 16 and 28 respectively. The 28 pin IC base is used for the microcontroller whereas the 16 pin IC base is used for the gates.

Battery Holder

The battery holder provides compartments to insert our power source which will be a DC battery.

IDE to program microcontroller

The IDE used to program the Atmega328P is Arduino IDE.

Android Studio to create the GATEway Companion App

Android Studio was used to design and build the companion app for GATEway which acts as a user interface.

\section{Methodology}

GATEway aims to identify the particular gate plugged in. The most absolute method of identifying a gate is through its truth table. Once the truth table is verified, there is no ambiguity left in verifying which gate it is.

The logics HIGH and LOW are applied to the gates and the corresponding outputs are compared with the truth tables. When the outputs are matched for all the four possible states, the gate is determined to be AND, OR, NOT, XOR, NAND or NOR. The determined IC information is relayed to the user interface which is an Android app via bluetooth.

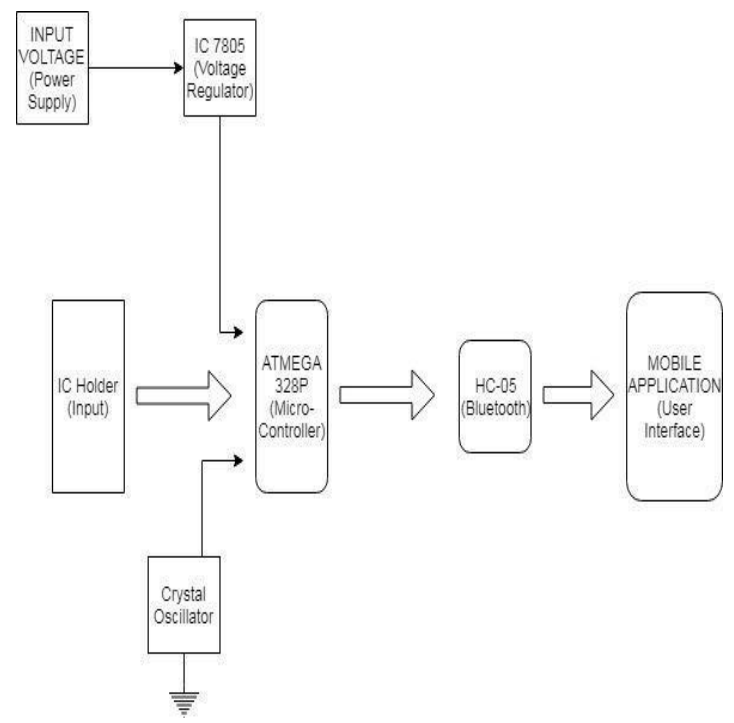

Figure 2: Block Diagram. 
Algorithm for detection of logic gate

1) Create the input truth table, which will form as the input for detection of the logic gate.

2) Check for NOR gate first, since pin number 1 is the output pin and pins 2 and 3 are inputs.

3) If NOR gate detection fails, check for NOT gate, since it has only one input.

4) If NOT gate detection fails, permute different inputs from the truth table and record the corresponding outputs.

5) The recorded outputs are then compared with the pre-defined outputs of the remaining basic gates, which are AND, OR, NAND and EXOR.

6) If no output is recorded after all these steps, it will mean that either the IC is faulty, or there is no IC at all. The above algorithm is based on the detection of only six basic logic gate ICs.

\section{The Companion App}

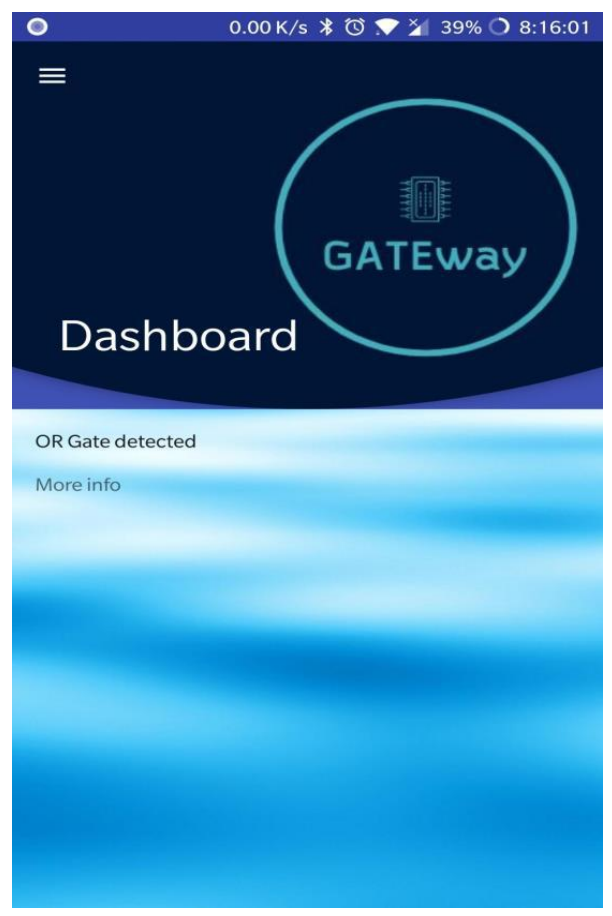

Figure 3: GATEway Companion App.

The GATEway Companion mobile application is avail-able for android devices, having Android version above 6.0 (Marshmallow).

Before launching the android application, make sure that the device bluetooth is turned and it is paired with "HC-05". Then insert the required IC in the GATEway device and observe the result on the mobile application.

\section{Result and Conclusion}

The app will display the gate detected once the gate in question is plugged in. The app will also provide a link to the datasheet of the gate detected.

\section{Future Scope}

The basic prototype designed includes the ability to detect the basic gate as well as provide supplementary documents such as the datasheet for the gate and tutorials. To develop the product further, we plan to include a solver that will output the solutions to boolean equations that will be fed in as input. The companion app will also display tutorials for solving of boolean equations for the user to develop their understanding further.

\section{References}

1. BASIC LOGIC GATES http://www.learnabout-electronics.org/Digital/dig21.php

2. SERIAL COMMUNICATION BETWEEN HC05 AND ANDROID DEVICE Saikat Patra and Prof Savitha.Pr, Reva University

3. Ritika Pahuja, Narender Kumar, Android Mobile Phone Controlled Bluetooth Robot Using 8051 Microcontroller, International Journal of Scientific Engineering and Research (IJSER) 
4. Hana R. Esmaeel, Apply Android Studio (SDK) Tools, International Journal of Advanced Research in Computer Science and Software Engineering

5. Yakov Shafranovich, Bluetooth Data Exchange Between Android Phones Without Pairing, Cornell University

6. Ming Yan and Hao Shi, SMART LIVING USING BLUETOOTH BASED ANDROID SMARTPHONE, International Journal of Wireless \& Mobile Networks (IJWMN) Vol. 5, No. 1, February 2013 